\title{
Modeling of Catalytic Reaction in Protein-Film Linear Scan Voltammetry at Rotating Disk Electrode
}

\author{
Milivoj Lovrić* \\ Department of Marine and Environmental Research, "Rudjer Bošković" Institute, P.O. Box \\ 180, HR-10002 Zagreb, Hrvatska (Croatia)
}

Received 9 March 2009; accepted 21 April 2009

\begin{abstract}
The numerical method for the simulation of linear scan voltammetry on the rotating disk electrode is adjusted to the problem of irreversible redox reaction between the adsorbed catalyst and the dissolved reactant under transient conditions. The response consists of the wave and the maximum. The peak current depends on the scan rate in linear scan voltammetry, while the limiting current of the wave depends on the rate of rotation of the working electrode. The rate constant of catalytic reaction is determined from the kinetic current under steady-state conditions.
\end{abstract}

Keywords: electro-catalytic reaction, rotating disk electrode, chemical reaction rate constant, linear scan voltammetry, protein-film voltammetry.

\section{Introduction}

The conversion of energy occurring in photosynthesis and respiration is realized through a complex sequence of electron transfer reactions $[1,2]$. The rate constants of these transfers are key parameters in the mentioned and several other biological processes ranging from cell defense to gene control [3 - 5]. Voltammetry is a simple and powerful method of investigating biologically relevant redox-active compounds [6 - 8]. For instance, by adsorption of the redox protein onto the surface of some suitable lipophilic electrode, the kinetics of transfer of electrons from the immobilized enzyme to dissolved substrate can be measured by cyclic voltammetry and chronoamperometry $[9,10]$. For sensors based on electro-catalytic reactions, the application of rotating disk electrodes (RDE) was proposed [11 - 13]. The sensors usually work under theoretically well described steady-state conditions [12], but for kinetic studies of catalytic effects the transient conditions are equally important [14]. In this communication the

\footnotetext{
* Corresponding author. E-mail address: mlovric@irb.hr
} 
numerical method for the simulation of linear scan voltammetry on the RDE [15] is adjusted to the problem of irreversible redox reaction between the adsorbed catalyst and the dissolved reactant under transient conditions. This reaction scheme belongs to the catalytic $\mathrm{EC}^{\prime}$ mechanism which was investigated on stationary electrodes under the conditions of various voltammetric methods [16 20].

\section{The model}

It is assumed that a certain redox couple is strongly adsorbed on the surface of RDE:

$$
A_{a d s} \leftrightarrow B_{a d s}^{n+}+n e^{-}
$$

The electrode reaction (1) is fast and reversible:

$$
\Gamma_{B}=\Gamma_{A} \exp (\varphi)
$$

where $\Gamma_{A}$ and $\Gamma_{B}$ are surface concentrations of the reactant and product, respectively, $\varphi=n F\left(E-E_{A / B}^{0}\right) / R T$ is dimensionless potential and $E_{A / B}^{0}$ is the standard potential. During the experiment, the sum of surface concentrations of the reactant and product does not change:

$$
\Gamma_{A}+\Gamma_{B}=\Gamma_{A}^{*}
$$

It is further assumed that in the solution there is a compound $Y$ which can be oxidized by the product of the surface redox reaction:

$$
B_{a d s}^{n+}+Y_{x=0} \rightarrow A_{a d s}+Z_{x=0}^{n+}
$$

In the investigated potential range the compound $Y$ can not be electro-oxidized on the bare electrode surface due to very slow electron transfer. The mass transport towards the surface of $\mathrm{RDE}$ is defined by the partial differential equation:

$$
\frac{\partial c_{Y}}{\partial t}=D \frac{\partial^{2} c_{Y}}{\partial x^{2}}+\kappa x^{2} \frac{\partial c_{Y}}{\partial x}
$$

and the boundary conditions:

$$
\begin{aligned}
& D\left(\frac{\partial c_{Y}}{\partial x}\right)_{x=0}=k_{f} \Gamma_{B} c_{Y, x=0} \\
& \frac{d \Gamma_{B}}{d t}=\frac{I}{n F S}-k_{f} \Gamma_{B} c_{Y, x=0}
\end{aligned}
$$

where $\kappa=0.51 \sqrt{\omega^{3} v^{-1}}$, while $\omega$ is the angular rotation rate of the disk, $v$ is the kinematic viscosity of the solution, $c_{Y}$ is the concentration of the compound $Y$, $D$ is the diffusion coefficient, $I$ is the current, $S$ is the electrode surface area, $n$ 
is the number of electrons, $F$ is Faraday constant and $k_{f}$ is the rate constant of irreversible reaction (4). Equation (5) is solved by Galerkin method of variable diffusion layer thickness [15]. It is assumed that the concentration profile is the linear function of the space coordinate between the electrode surface $(x=0)$ and a certain distance $\delta$ which is the function of time:

$$
\begin{array}{ll}
c_{Y}(x, t)=c_{Y, x=0}+\left(c_{Y}^{*}-c_{Y, x=0}\right) \frac{x}{\delta}, & \text { for } 0 \leq x \leq \delta \\
c_{Y}(x, t)=c_{Y}^{*}, \quad \quad \text { for } x>\delta &
\end{array}
$$

where $c_{Y}^{*}$ is the bulk concentration of $Y$. Equation (5) is integrated from $x=0$ to infinity:

$$
\int_{0}^{\infty} \frac{\partial c_{Y}}{\partial t} d x=-D\left(\frac{\partial c_{Y}}{\partial x}\right)_{x=0}+\kappa \int_{0}^{\infty} x^{2} \frac{\partial c_{Y}}{\partial x} d x
$$

and by using the assumed gradients for $0 \leq x \leq \delta$ :

$$
\begin{aligned}
& \frac{\partial c_{Y}}{\partial t}=\left(1-\frac{x}{\delta}\right) \frac{d c_{Y, x=0}}{d t}-\left(c_{Y}^{*}-c_{Y, x=0}\right) \frac{x}{\delta^{2}} \frac{d \delta}{d t} \\
& \frac{\partial c_{Y}}{\partial x}=\frac{c_{Y}^{*}-c_{Y, x=0}}{\delta}
\end{aligned}
$$

and for $x>\delta$ :

$$
\frac{\partial c_{Y}}{\partial t}=\frac{\partial c_{Y}}{\partial x}=0
$$

it is transformed into the differential equation on time:

$$
\frac{d y}{d t}=4 D-\frac{4}{3} \kappa \sqrt{y^{3}}+\frac{2 y}{c_{Y}^{*}-c_{Y, x=0}} \frac{d c_{Y, x=0}}{d t}
$$

where $y=\delta^{2}$. Under steady-state conditions:

$$
\frac{d y}{d t}=\frac{d c_{Y, x=0}}{d t}=c_{Y, x=0}=0
$$

the solution of equation (14) is:

$$
\delta_{s s}=\left(\frac{3 D}{\kappa}\right)^{\frac{1}{3}}
$$

which is the steady-state diffusion layer thickness. A general solution of eq. (14) can be obtained by the numerical integration. By combining equations (6) and (12) one obtains:

$$
c_{Y, x=0}=\frac{c_{Y}^{*} D}{D+k_{f} \Gamma_{B} \delta}
$$


Also, the combination of eqs. (2) and (3) gives the following relationship:

$$
\Gamma_{B}=\frac{\Gamma_{A}^{*} \exp (\varphi)}{1+\exp (\varphi)}
$$

In linear scan voltammetry the derivations of $c_{Y, x=0}$ and $\Gamma_{B}$ on time are:

$$
\begin{aligned}
& \frac{d c_{Y, x=0}}{d t}=-\frac{c_{Y}^{*} k_{f} D}{\left(D+k_{f} \delta \Gamma_{B}\right)^{2}}\left(\delta \frac{d \Gamma_{B}}{d t}+\Gamma_{B} \frac{d \delta}{d t}\right) \\
& \frac{d \Gamma_{B}}{d t}=\frac{\frac{n F}{R T} \frac{d E}{d t} \Gamma_{A}^{*} \exp (\varphi)}{[1+\exp (\varphi)]^{2}}
\end{aligned}
$$

Using the approximation:

$$
\frac{d y}{d t} \approx \frac{\Delta y}{\Delta t}=\frac{y_{j+1}-y_{j}}{\Delta t}
$$

equation (14) is transformed into the system of recursive formulae:

$$
\begin{gathered}
y_{1}=2 D \Delta t \\
y_{j+1}=y_{j}+\frac{D+D \exp \left(\varphi_{j}\right)+k_{f} \Gamma_{A}^{*} \sqrt{y_{j}} \exp \left(\varphi_{j}\right)}{2 D+2 D \exp \left(\varphi_{j}\right)+k_{f} \Gamma_{A}^{*} \sqrt{y_{j}} \exp \left(\varphi_{j}\right)}\left[4 D \Delta t-\frac{4}{3} \kappa \Delta t \sqrt{y_{j}^{3}}\right] \\
-\frac{2 y_{j} D \Delta t \frac{n F}{R T} \frac{d E}{d t}}{2 D+2 D \exp \left(\varphi_{j}\right)+k_{f} \Gamma_{A}^{*} \sqrt{y_{j}} \exp \left(\varphi_{j}\right)} \\
\varphi_{j}=\frac{n F}{R T}\left(E_{s t}+\frac{d E}{d t} j \Delta t-E_{A / B}^{0}\right) \\
\delta_{j}=+\sqrt{y_{j}}
\end{gathered}
$$

The current is defined by eq. (7):

$$
\begin{gathered}
\frac{I_{j}}{I_{d i f}}=\frac{\frac{n F}{R T} \frac{d E}{d t} \Gamma_{A}^{*} \delta_{s s} \exp \left(\varphi_{j}\right)}{D c_{Y}^{*}\left[1+\exp \left(\varphi_{j}\right)\right]^{2}}+\frac{k_{f} \Gamma_{A}^{*} \delta_{s s} \exp \left(\varphi_{j}\right)}{D\left[1+\exp \left(\varphi_{j}\right)\right]+k_{f} \Gamma_{A}^{*} \delta_{j} \exp \left(\varphi_{j}\right)} \\
I_{d i f}=n F S \frac{D c_{Y}^{*}}{\delta_{s s}}
\end{gathered}
$$

Equations (26) and (27) are obtained by combining eq. (7) with eqs. (17), (18) and (20). The first term on the right-hand side of eq. (26) is peak-shaped. It tends to zero if both $E<<E_{A / B}^{0}$ and $E>E_{A / B}^{0}$. Equation (18) shows that $\Gamma_{B}=\Gamma_{A}^{*}$ if $E>>E_{A / B}^{0}$. So, if $E>>E_{A / B}^{0}$, the limiting kinetic current appears: 


$$
\lim _{E>E_{A / B}^{0}}\left(\frac{I}{n F S}\right)=\frac{k_{f} \Gamma_{A}^{*} c_{Y}^{*} D}{D+k_{f} \Gamma_{A}^{*} \delta_{s S}}
$$

Equation (27) is the limiting value of eq. (28) if $D<<k_{f} \Gamma_{A}^{*} \delta_{s s}$.

The simulation was performed using constant values of diffusion coefficient and kinematic viscosity, while the rotation rate of $\mathrm{RDE}$ and the scan rate in CV were varied.

\section{Results and discussion}

Electro-catalytic oxidation of the compound $Y$ on RDE depends on the product of the oxidation rate constant and the surface concentration of the catalyst $\left(k_{f} \Gamma_{A}^{*}\right)$ and on the ratio of the latter and the bulk concentration of the reactant $\left(\Gamma_{A}^{*} / c_{Y}^{*}\right)$. Fig. 1 shows simulated cyclic voltammograms of coupled reactions (1) and (4) on $\mathrm{RDE}$, for various products $k_{f} \Gamma_{A}^{*}$ and the constant ratio $\Gamma_{A}^{*} / c_{Y}^{*}$. The current is made dimensionless by dividing with the diffusion steady-state current defined by eq. (27). Voltammograms consist of the wave and the peak. The latter originates from the surface electrode reaction (1). The net peak current depends on the ratio $\Gamma_{A}^{*} / c_{Y}^{*}$ and does not change with the variation of the product $k_{f} \Gamma_{A}^{*}$.

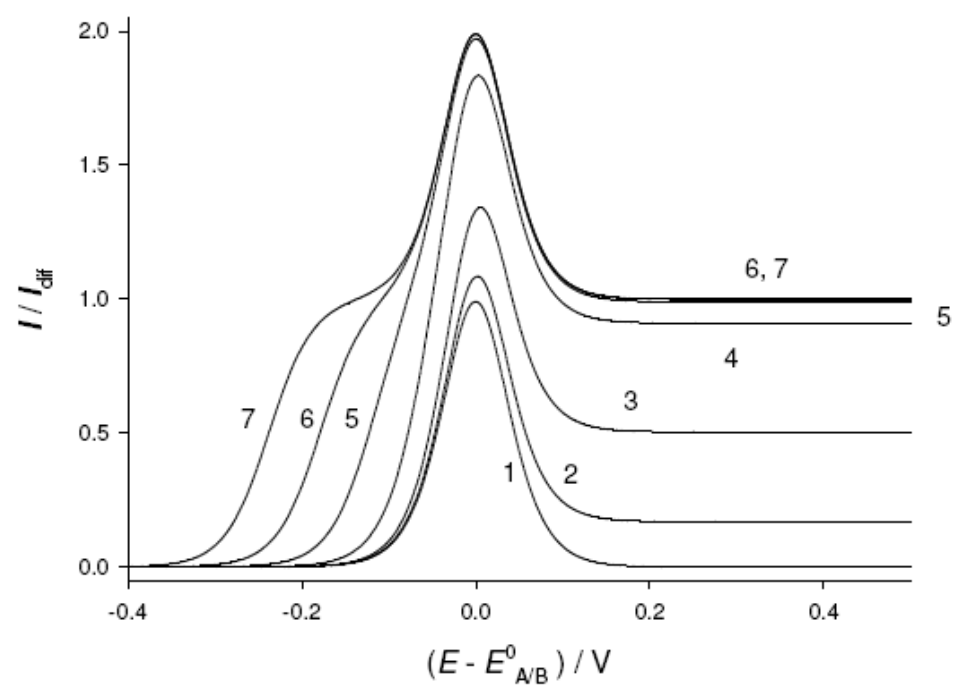

Figure 1. Dimensionless LSV of electro-catalytic oxidation of $Y$ on RDE. $D=10^{-5}$ $\mathrm{cm}^{2} / \mathrm{s}, v=10^{-2} \mathrm{~cm}^{2} / \mathrm{s}, n=1, \omega=100 \pi \mathrm{s}^{-1}, d E / d t=0.1 \mathrm{~V} / \mathrm{s}, \Gamma_{A}^{*} / c_{Y}^{*}=0.01 \mathrm{~cm}$ and $k_{f} \Gamma_{A}^{*} / \mathrm{cm} \mathrm{s}^{-1}=0$ (1), 0.002 (2), 0.01 (3), 0.1 (4), 1 (5), 10 (6) and 100 (7).

Fig. 2 shows the maximum and steady-state currents of voltammograms as functions of the logarithm of $k_{f} \Gamma_{A}^{*}$ product. The curve 1 in this figure shows that $I_{\max }=I_{0.5 V}+0.9916 I_{d i f}$, where $I_{\max } / I_{d i f}=0.9916$ is the maximum of the curve 1 in Fig. 1 and $I_{0.5 V} / I_{d i f}$ is the dimensionless current corresponding to the potential difference $E-E_{A / B}^{0}=0.5 \mathrm{~V}$. The potential of maximum is equal to the standard 
potential if either $\log \left(k_{f} \Gamma_{A}^{*}\right)<-3$, or $\log \left(k_{f} \Gamma_{A}^{*}\right)>0$. Within these boundaries, the peak potential increases to $E_{\max }-E_{A / B}^{0}=0.006 \mathrm{~V}$ at $k_{f} \Gamma_{A}^{*}=0.02 \mathrm{~cm} / \mathrm{s}$. The steady-state currents are limiting currents of the waves. They change from zero to one, as can be seen in Fig. 2. This component of the response originates from the catalytic oxidation of the compound $Y$. Considering that in Fig. $1 D=10^{-5} \mathrm{~cm}^{2} / \mathrm{s}$ and $\delta_{s s}=1.0185 \times 10^{-3} \mathrm{~cm}$, the limiting value $I_{\text {lim }}=I_{d i f}$ is achieved if $k_{f} \Gamma_{A}^{*}>1$, which satisfies the condition $D<<k_{f} \Gamma_{A}^{*} \delta_{s s}$ (see eq. 28).

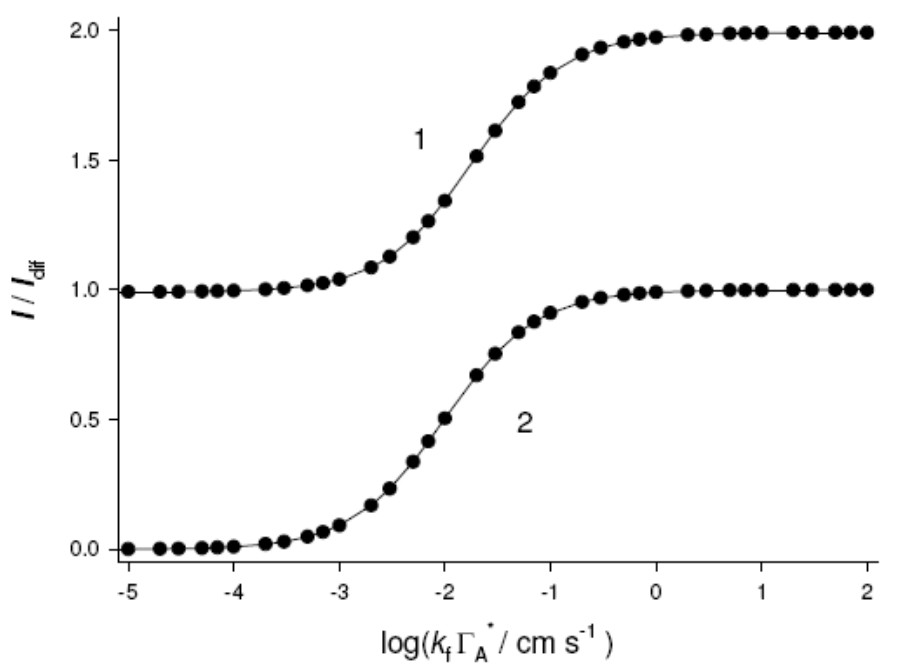

Figure 2. Dependence of the maximum ( $I_{\max } / I_{d i f}$; marked as 1) and the steady-state ( $I_{0.5 \mathrm{~V}} / I_{\text {dif }}$; marked as 2) dimensionless $\mathrm{CV}$ currents of $Y$ oxidation on the logarithm of the product $k_{f} \Gamma_{A}^{*}$. All parameters are as in Fig. 1.

The half-wave potentials of the wave-components of the voltammograms in Fig. 1 satisfy the relationship: $E_{1 / 2}-E_{\mathrm{A} / \mathrm{B}}^{0}=-0.059 \times \log \left(k_{f} \Gamma_{A}^{*}\right)-0.120 \mathrm{~V}$, if $k_{f} \Gamma_{A}^{*} \geq 1$ and $n=1$. This potential can be calculated for steady-state conditions using equations (26) - (28):

$$
\frac{k_{f} c_{Y}^{*} \Gamma_{A}^{*} D \exp \left(\varphi_{1 / 2}\right)}{D+\left(D+k_{f} \delta_{s s} \Gamma_{A}^{*}\right) \exp \left(\varphi_{1 / 2}\right)}=\frac{k_{f} c_{Y}^{*} \Gamma_{A}^{*} D}{2\left(D+k_{f} \delta_{s s} \Gamma_{A}^{*}\right)}
$$

where $\varphi_{1 / 2}=n F\left(E_{1 / 2}-E_{A / B}^{0}\right) / R T$. The solution is:

$$
E_{1 / 2}-E_{A / B}^{0}=\frac{R T}{n F} \ln \frac{D}{D+k_{f} \delta_{s s} \Gamma_{A}^{*}}
$$

If $k_{f} \Gamma_{A}^{*}=0, E_{1 / 2}=E_{A / B}^{0}$ and if $k_{f} \Gamma_{A}^{*} \delta_{s s} \gg D$ the half-potential is:

$$
E_{1 / 2}-E_{A / B}^{0}=\frac{R T}{n F} \ln \frac{D}{\delta_{s s}}-\frac{R T}{n F} \ln \left(k_{f} \Gamma_{A}^{*}\right)
$$


This shows that very fast chemical reaction consumes the product of electrode reaction and shifts the oxidation wave towards lower potentials. The half-wave potential of the curve 7 in Fig. 1 is $E_{1 / 2}-E_{A / B}^{0}=-0.237 \mathrm{~V}$. In this curve it can be noted that the peak component developes on the plateau of the wave component. The potential range within which this occurs can be calculated using equation (26):

$$
\frac{k_{f} \Gamma_{A}^{*} \delta_{s s} \exp (\varphi)}{D+\left(D+k_{f} \Gamma_{A}^{*} \delta_{s s}\right) \exp (\varphi)}>0.95
$$

For the curve 7 in Fig. 1 the solution is:

$$
E-E_{A / B}^{0}>-0.161 \mathrm{~V}
$$

The second condition is:

$$
\frac{\frac{n F}{R T} \frac{d E}{d t} \delta_{s s} \Gamma_{A}^{*} \exp (\varphi)}{D c_{Y}^{*}(1+\exp (\varphi))^{2}}<0.05
$$

For the same curve the solutions are:

$$
\begin{aligned}
& E-E_{A / B}^{0}<-0.112 \mathrm{~V} \\
& E-E_{A / B}^{*}>0.112 \mathrm{~V}
\end{aligned}
$$

So, between $-0.161 \mathrm{~V}$ and $-0.112 \mathrm{~V}$ the wave component stagnates and the peak component developes.

Because of constant ratio $\Gamma_{A}^{*} / c_{Y}^{*}$, voltammograms shown in Fig. 1 correspond to various oxidation reactions (4), each with the different rate constant $k_{f}$. However, the variation of the product $k_{f} \Gamma_{A}^{*}$ can be achieved by the variation of the surface concentration of the catalyst, but in this case the ratio $\Gamma_{A}^{*} / c_{Y}^{*}$ must be also changed. An example is shown in Fig. 3. The main difference between Figs. 1 and 3 is the change of the net peak current because of the change of the ratio $\Gamma_{A}^{*} / c_{Y}^{*}$ in Fig. 3. The curve 6 in Fig. 3 and the curve 4 in Fig. 1 are identical because all parameters are equal in both figures, but the curve 1 in Fig. 3 is significantly different from the curve 3 in Fig. 1 because $\Gamma_{A}^{*} / c_{Y}^{*}$ is $0.001 \mathrm{~cm}$ in Fig. 3 and $0.01 \mathrm{~cm}$ in Fig. 1. The dimensionless steady-state current of the curve 1 in Fig. 3 is equal to the dimensionless steady-state current of the curve 3 in Fig. 1 because the second term on the right-hand side of eq. (26) does not depend on the ratio $\Gamma_{A}^{*} / c_{Y}^{*}$.

For analytical purposes, the relationships between either transient, or steady-state current and the bulk concentration of the compound $Y$ are important. Fig. 4 shows that the latter relationship is linear: $I_{0.5 V} / n F S=8.941 \times 10^{-3} c_{Y}^{*}$ (see curve 1). The slope of this straight line is defined by eq. (28). In Fig. 3 it can be seen that the ratio of the maximum and steady-state currents decreases as the ratio 
$\Gamma_{A}^{*} / c_{Y}^{*}$ is diminished. Consequently, the maximum current depends on $c_{Y}^{*}$ nonlinearly, starting from $I_{\max }=n F S(n F / R T)(d E / d t) \Gamma_{A}^{*} / 4$, for $c_{Y}^{*}=0$, and approaching the linear relationship asymptotically (see curve 2 in Fig. 4). Using the catalytic oxidation on RDE, the compound $Y$ can be determined in the concentrations higher than $10^{-5} \mathrm{M}$. In the presence of several electroactive substances, the peak current rather than the limiting current can be used for the analysis of the compound $Y$.

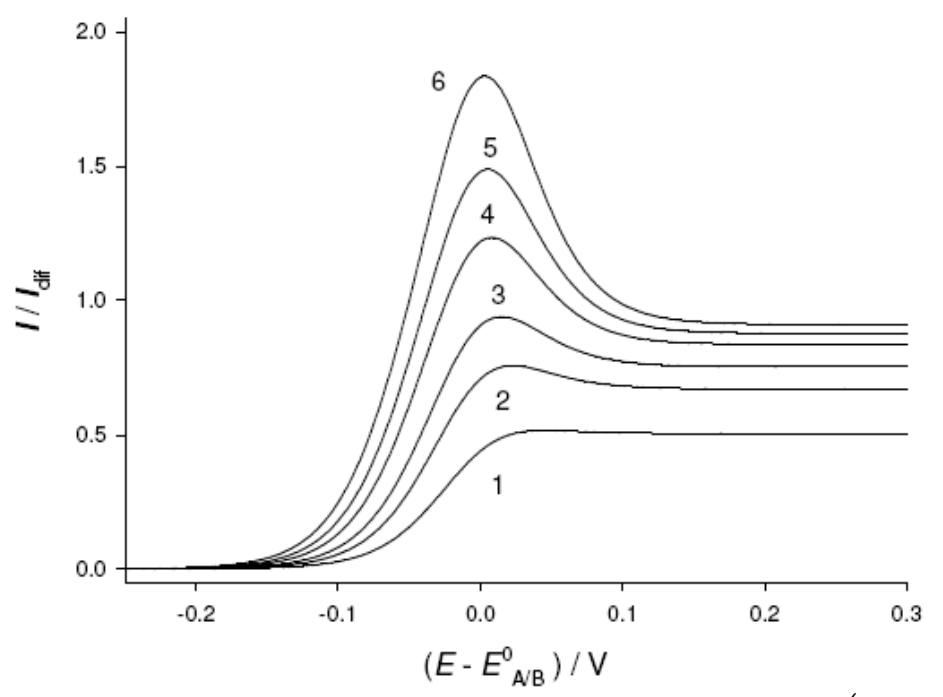

Figure 3. Dimensionless LSV of coupled reactions (1) and (4). $\left(\Gamma_{A}^{*} / c_{Y}^{*}\right) / \mathrm{cm}=0.001$ (1), 0.002 (2), 0.003 (3), 0.005 (4), 0.007 (5) and 0.01 (6); $k_{f} \Gamma_{A}^{*} / \mathrm{cm} \mathrm{s}^{-1}=0.01$ (1), 0.02 (2), 0.03 (3), 0.05 (4), 0.07 (5) and 0.1 (6). All other parameters are as in Fig. 1.

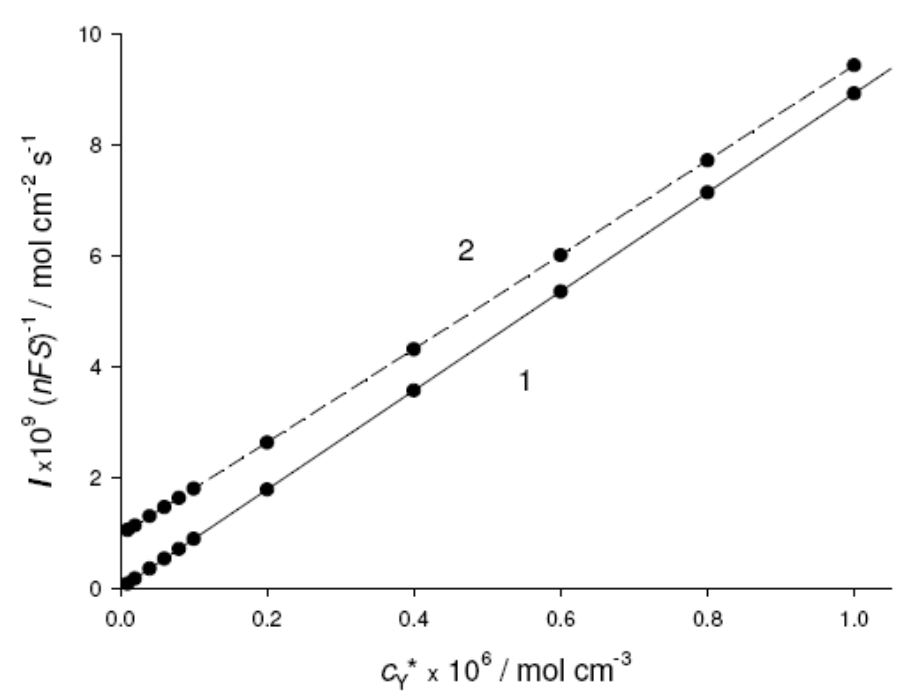

Figure 4. Dependence of normalized steady-state (1) and maximum (2) currents on the bulk concentration of compound $Y . \Gamma_{A}^{*}=10^{-9} \mathrm{~mol} \mathrm{~cm}^{-2}$ and $k_{f}=10^{8} \mathrm{~cm}^{3} \mathrm{~s}^{-1} \mathrm{~mol}^{-1}$. All other parameters are as in Fig. 1. 
The investigated responses consist of the transient part, which is the rising portion of the wave and the whole maximum, and of the steady-state part, which is the limiting current of the wave. The scan rate in LSV can influence only the transient part, while the rotation rate of RDE influences only the steady-state part. The dependence of dimensionless maximum current on the scan rate is shown in Fig. 5. It is a straight line if $d E / d t>30 \mathrm{mV} / \mathrm{s}$. At the lowest scan rates the maximum current tends to the steady-state current, which is $0.5046 \times I_{\text {dif }}$ for the assumed experimental parameters. For this reason the net peak current $\left(I_{\max }-I_{0.5 \mathrm{~V}}\right)$ is a linear function of scan rate if $d E / d t>15 \mathrm{mV} / \mathrm{s}$, and tends to zero below this boundary.

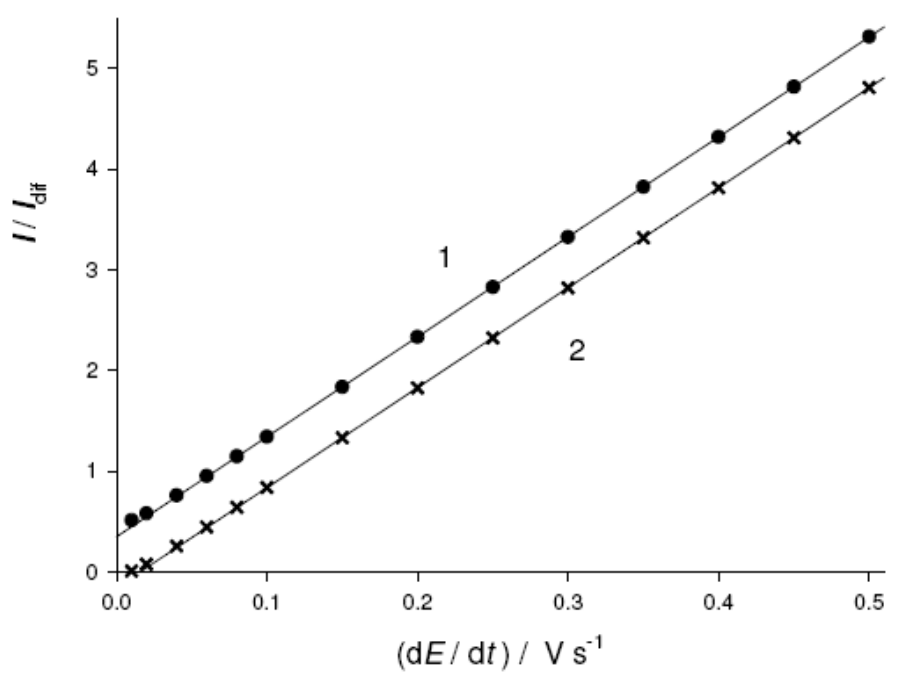

Figure 5. Dependence of $I_{\max } / I_{d i f}(1)$ and $\left(I_{\max }-I_{0.5 \mathrm{~V}}\right) / I_{d i f}(2)$ on the scan rate. $k_{f} \Gamma_{A}^{*}$ $=0.01 \mathrm{~cm} \mathrm{~s}^{-1}$ and all other parameters are as in Fig. 1 .

The relationship between the steady-state current and the rate of rotation of RDE is defined by eqs. (28) and (16). It can be transformed into linear dependence of reciprocal of current on the inverse value of square-root of the rotation rate:

$$
\frac{n F S}{I_{\lim }}=\frac{1}{k_{f} \Gamma_{A}^{*} c_{Y}^{*}}+\frac{1.805 \times v^{1 / 6}}{c_{Y}^{*} D^{2 / 3}} \frac{1}{\sqrt{\omega}}
$$

This dependence is shown in Fig. 6, for three different values of the product $k_{f} \Gamma_{A}^{*}$. The intercepts of these straight lines serve for the determination of the oxidation rate constants. The surface concentration of the catalyst $\Gamma_{A}^{*}$ can be determined from the peak current $I_{\max }$ in the absence of the compound $Y$ $\left(c_{Y}^{*}=0\right.$; see the intercept of curve 2 in Fig. 4). Hence, the advantage of RDE is in the establishment of steady-state conditions under which the kinetic current can be analyzed according to equation (37). 


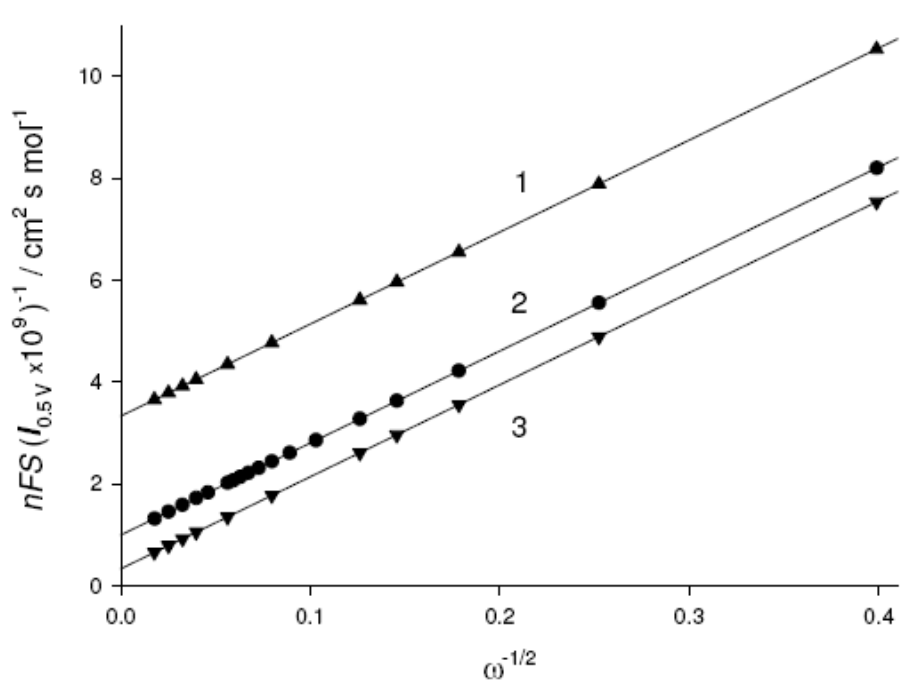

Figure 6. Dependence of normalized reciprocal of steady-state current on the inverse value of square-root of RDE rotation rate. $\Gamma_{A}^{*}=10^{-9} \mathrm{~mol} \mathrm{~cm}^{-2}, c_{Y}^{*}=10^{-7} \mathrm{~mol} \mathrm{~cm}^{-3}$ and $k_{f} / \mathrm{cm}^{3} \mathrm{~s}^{-1} \mathrm{~mol}^{-1}=3 \times 10^{6}(1), 10^{7}(2)$ and $3 \times 10^{7}$ (3). All other parameters are as in Fig. 1.

\section{Conclusion}

The model described in this paper is an adequate representation of protein-film voltammetric experiments [6]. Some examples of electro-catalytic reactions considered here are the oxidation of glucose by glucose oxidase adsorbed on the electrode surface [21] and the oxidation of dissolved succinate to fumarate catalyzed by succinate dehydrogenase adsorbed on the rotating disk electrode [22]. In linear scan voltammetry on RDE the theoretical response of this type of reactions can be calculated by the proposed numerical method. It is developed for the first, transient part of the response, in which the diffusion layer thickness is time-depending and the concentration of the reactant at the working electrode surface depends on both the electrode potential and the oxidation rate constant. The steady-state part of the response appears as a special case of the general solution. Finally, it is shown that for both kinetic and analytical measurements the steady-state conditions are the most appropriate.

\section{Acknowledgement}

Valuable contributions by the referees and the financial support by the Croatian Ministry of Science, Education and Sports are gratefully acknowledged.

\section{References}

1. F.A. Armstrong, Bioelectrochemistry of Biomacromolecules, Birkhauser, Basel, 1997.

2. A.G. Volkov, M.I. Volkova-Gugeshashvili, C.L. Brown-McGauley, A.J. Osei, Electrochim. Acta 52 (2007) 2905-2912.

3. A.K. Satpati, M. Kumbhakar, S. Nath, H. Pal, J. Photochem. Photobiol. A: Chem. 200 (2008) 270-276. 
4. M.F. Chaplin, C. Bucke, Enzyme Technology, Cambridge University Press, Cambridge, 1990.

5. C. Leger, S.J. Elliott, K.R. Hoke, L.J.C. Jeuken, A.K. Jones, F.A. Armstrong, Biochem. 42 (2003) 8653-8662.

6. F.A. Armstrong, H.A. Heering, J. Hirst, Chem. Soc. Rev. 26 (1997) 169179.

7. F.A. Armstrong, G.S. Wilson, Electrochim. Acta 45 (2000) 2623-2645.

8. A. Heller, Acc. Chem. Res. 23 (1990) 128-134.

9. $\quad$ N.F. Hu, Pure App. Chem. 73 (2001) 1979-1991.

10. Y.H. Wu, S.S. Hu, Microchim. Acta 159 (2007) 1-17.

11. N. Oyama, K. Sato, H. Matsuda, J. Electroanal. Chem. 115 (1980) 149155.

12. C.P. Andrieux, J.M. Dumas-Bouchiat, J.M. Saveant, J. Electroanal. Chem. 123 (1981) 171-187.

13. T. Ikeda, C.R. Leidner, R.W. Murray, J. Electroanal. Chem. 138 (1982) 343-365.

14. J.M. Saveant, E. Vianello, Electrochim. Acta 10 (1965) 905-920.

15. M. Lovrić, J. Osteryoung, J. Electroanal. Chem. 197 (1986) 63-75.

16. J.M. Saveant, E. Vianello, Electrochim. Acta 12 (1967) 629-646.

17. A.J. Bard, L. Faulkner, Electrochemical Methods, $2^{\text {nd }}$ edn, Wiley, New York, 2001, pp 471-533.

18. F. Marken, A. Neudeck, A.M. Bond, in F. Scholz (ed.), Electroanalytical Methods, Springer, Berlin, 2002, pp 51-97.

19. Š. Komorsky-Lovrić, M. Lovrić, in E.P. Vargus (ed.), New Research in Electrochemistry, Nova Sci. Publ., New York, 2007, pp 1-44.

20. R.G. Compton, C.E. Banks, Understanding Voltammetry, World Sci. Publ., New Jersey, 2007, pp 266-270.

21. W.J. Albery, P.N. Bartlett, D.H. Craston, J. Electroanal. Chem. 194 (1985) 223-235.

22. J. Hirst, A. Sucheta, B.A.C. Ackrell, F.A. Armstrong, J. Am. Chem. Soc. 118 (1996) 5031-5038. 